\title{
Effect of ankle joint fixation on tibialis anterior muscle activity during split-belt treadmill walking in healthy subjects: A pilot study
}

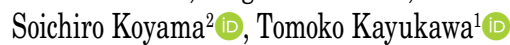 \\ ${ }^{1}$ Department of Rehabilitation, Fujita Health University Bantane Hospital, Nagoya, Japan \\ ${ }^{2}$ Faculty of Rehabilitation, School of Health Sciences, Fujita Health University, Toyoake, Japan \\ ${ }^{3}$ Department of Rehabilitation Medicine I, Fujita Health University, Toyoake, Japan \\ ${ }^{4}$ Department of Rehabilitation, Fujita Health University Hospital, Toyoake, Japan
}

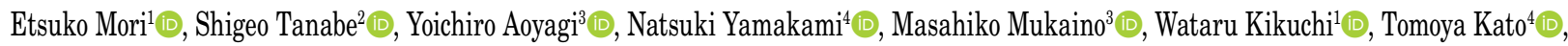

Received: July 02, 2019 Accepted: December 08, 2019 Published online: March 04, 2021

\begin{abstract}
Objectives: This study aims to examine the characteristics of muscle activity change of the tibialis anterior (TA) muscle in healthy adults while they walked on a split-belt treadmill with one fixed ankle.

Patients and methods: This randomized controlled trial was conducted between November 2017 and July 2018. Fourteen healthy male individuals (mean age 31.4 years; range, 23 to 50 years) were divided into two groups: right ankle joint fixed by ankle-foot orthosis (fixation group) and no orthosis (control group). Both groups were asked to walk on a treadmill with the same belt speed. After familiarizing with walking on both belts at $5.0 \mathrm{~km} / \mathrm{h}$, they walked for 6 min with the right belt slower $(2.5 \mathrm{~km} / \mathrm{h})$ and the left faster $(5.0 \mathrm{~km} / \mathrm{h})$. For analysis, the 6 min were divided equally among three time periods. The TA muscle activity was calculated at first and last time periods. We compared muscle activities in time periods (early and late phase) and in groups (fixation and control) using two-way mixed analysis of variance.
\end{abstract}

Results: The TA muscle activity decreased in the late phase regardless of ankle joint fixation, and also decreased in the fixation group regardless of the time periods. There was an interaction between these factors.

Conclusion: These data show that changes in the TA muscle activity were smaller in the fixation group, suggesting that the ankle joint fixation reduces the adaptation.

Keywords: Ankle joint, gait, orthosis, walking.

Approximately 220,000 people have strokes per year in Japan. ${ }^{[1]}$ Walking disorder after stroke is a factor that reduces mobility capability and restricts range of activity. ${ }^{[2]}$ Improvement of walking is an important therapeutic objective of rehabilitation medicine after stroke. In the stroke treatment guidelines, it is recommended to use a treadmill as rehabilitation of gait disturbance. ${ }^{[3-7]}$

The asymmetry characteristics of hemiplegic gait are caused by dysfunctions such as motor paralysis and spasticity, ${ }^{[8-12]}$ and affects walking speed. ${ }^{[13]}$
Asymmetry is a factor affecting daily activities and quality of life. ${ }^{[2]}$ Results of subsequent studies have shown that exercise can improve asymmetry, ${ }^{[3-5]}$ and the use of special treadmills with left and right belts, which can run at different speeds, has attracted attention in recent years owing to its effectiveness. ${ }^{[14-16]}$ A study showed that step length symmetry improved after gait exercise as an adaptation to split-belt treadmill intervention in patients with hemiplegia. ${ }^{[14]}$ Other studies have reported on its long-term effects. ${ }^{[15,16]}$ Specifically, in 
cases of paralyzed walking with a longer step on the paretic leg, the asymmetry is exaggerated by slowing the paralyzed side belt. The exaggerated asymmetry decreases over time as a result of adaptation and returns to the original degree of asymmetry. When the belt speeds are returned to the same speed, the changed gait pattern after adaptation remains, and thus, the symmetry of stride is improved compared with that before the intervention. ${ }^{[14,15]}$

Foot stiffness at heel contact is important for adaptation to split-belt treadmill exercise, the tibialis anterior (TA), particularly, plays a major role; ${ }^{[17]}$ in healthy subjects, the activity of TA on the slow belt side during the stance phase (particularly, heel contact) increased during the initial adaptation phase, and thus, TA activity decreased according to the adaptation. Generally, at the initial stance (0-12\% gait cycle), the heel locker consists of TA eccentric contraction, ${ }^{[18]}$ which inhibits dropping of the foot and plays an important role in shock absorption.

Conversely, in hemiplegia walking, usage of an ankle-foot orthosis compensates for the function of dorsiflexion eccentric contraction. ${ }^{[19]}$ Many hemiplegic stroke patients use ankle-foot orthosis, but there have been no reports focusing on activity changes of the TA muscle with a fixed ankle while walking on a split-belt treadmill. Herein, we hypothesized that the change in muscle activity of the TA muscle is smaller when the ankle was fixed. Therefore, in this study, we aimed to examine the characteristics of muscle activity change of the TA muscle in healthy adults while they walked on a split-belt treadmill with one fixed ankle.

\section{PATIENTS AND METHODS}

This randomized controlled trial study was conducted at Fujita Health University Bantane Hospital between November 2017 and July 2018. Fourteen healthy adult males (mean age 31.4 years; range, 23 to 50 years) with no reported heart disease, stroke, orthopedic history, or disease participated in this study. No participants had any habituated physical activity (e.g. playing sports, muscle training, and running). Participants were recruited by means of advertisements and randomly allocated into two groups based on the sequential order of sampling; the first half were the fixation group and the other half were the control group. In the first group $(n=7)$, their right ankle joint was fixed by an ankle-foot orthosis. In the other group $(n=7)$, they did not wear orthoses. The study protocol was approved by the
Ethics Committee of Fujita Health University (no. HM16-264). A written informed consent was obtained from each participant. The study was conducted in accordance with the principles of the Declaration of Helsinki.

The TA muscle activity was recorded using BIMUTAS (Kissei Comtec, Co., Ltd., Nagano, Japan). Prior to walking, we measured each subject's maximum voluntary isometric contraction (MVIC) of TA muscle for $3 \mathrm{sec}$. The posture during measurement was referred to as normal (Grade 5) by the manual muscle testing method. ${ }^{[20]}$

The split-belt treadmill used in the study was a low-deck treadmill (Ohtake Root Kogyo Co., Ltd., Iwate, Japan), which is capable of controlling different speeds on the left and right belts. To identify the gait cycle in treadmill walking, we used a threedimensional (3D) motion capture system (Kinema Tracer System; Kissei Comtec, Co., Ltd., Nagano, Japan). Participants walked on the treadmill with hemispherical markers and surface electromyography (EMG). Previous work shows that walking adaptation is seen with a split-belt treadmill after $6 \mathrm{~min}^{\left[{ }^{[2]]}\right.}$ this effect was verified in both healthy individuals and hemiplegic patients by setting the belt speed of one belt to $1 / 2$ slower. ${ }^{[15,22]}$ In this study, individuals in both groups walked for the first 2 min with both belts at $5.0 \mathrm{~km} / \mathrm{h}$ for familiarizing themselves with walking on a treadmill. For the next $6 \mathrm{~min}$ of walking, the right belt was slowed to $2.5 \mathrm{~km} / \mathrm{h}$, and the left belt was maintained at $5.0 \mathrm{~km} / \mathrm{h}$ (Figure 1).

The fixation group walked at low speed with metal double upright ankle-foot orthosis on the right leg. The ankle angle of the orthosis was fixed at 0 degrees. During treadmill walking, participants were not given special instructions on walking style. Each participant wore a trunk belt suspended from the ceiling to prevent falls during the study.

Walking data were recorded for $15 \mathrm{sec}$ in the phase of split-belt walk just after the transition to split-belt (early phase) and after $4 \mathrm{~min}$ (late phase). Electromyographic data and 3D motion analysis data were synchronized by recording trigger signals.

The TA muscle activity on the right (slow belt) was recorded by bipolar montage. We shaved and wiped the skin with alcohol and sandpaper to prepare the skin for the electrode. Electrodes were placed on the midportion of the muscle belly and were aligned along the long axis of the muscle. During walking, the lead wires were fixed to the body to eliminate 


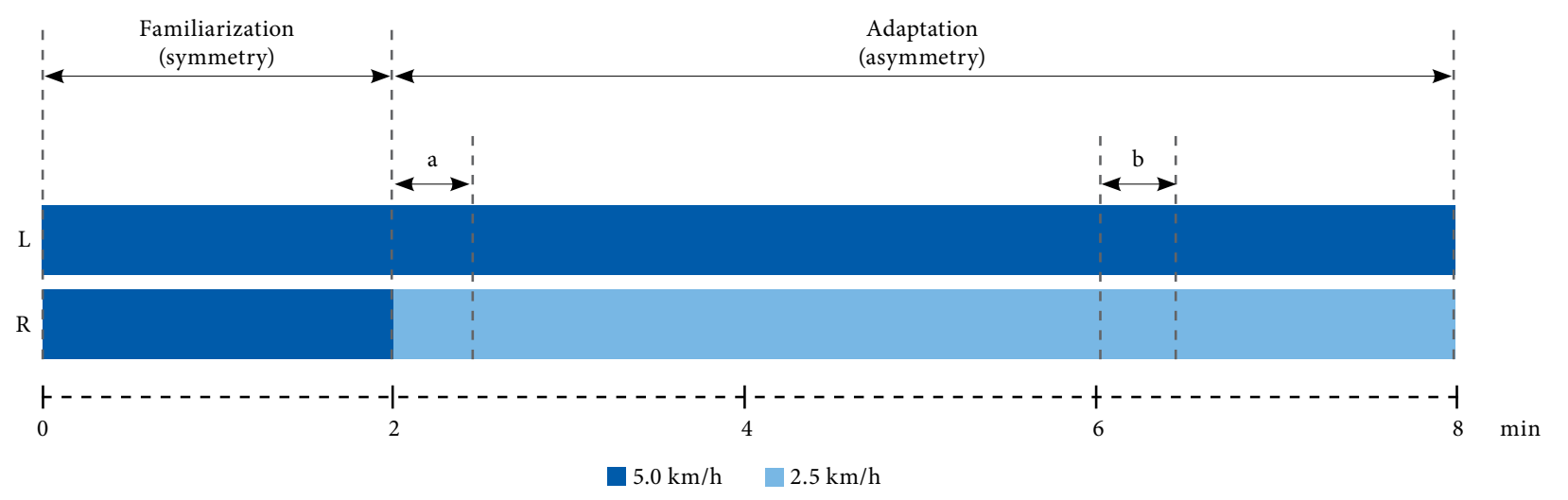

Figure 1. Time course of experimental protocol.

Upper and lower bars show left and right belt speeds, respectively. Subjects were asked to walk at $5 \mathrm{~km} / \mathrm{h}$ in symmetric condition for $2 \mathrm{~min}$ for familiarization. Subsequently, right belt speed was changed to $2.5 \mathrm{~km} / \mathrm{h}$ for $6 \mathrm{~min}$ as an adaptation period. Data were recorded for 15 sec at two instances: just after belt speed changed (a: early phase), and $4 \mathrm{~min}$ after belt speed changed (b: late phase).

artifacts caused by shaking of the electrode lead wires. We used a myoelectric amplifier to record electromyographic data (WBE-5000 manufactured by Nihon kohden Co., Ltd., Tokyo, Japan), applied a ham filter to remove alternating current interference from the power supply and devices. The sampling frequency and bandpass filter were set to $2,000 \mathrm{~Hz}$ and $30-500 \mathrm{~Hz}$.

Optical markers of $30 \mathrm{~mm}$ were placed on the knee joint at the midpoint of lateral epicondyle of the femur and the lateral malleolus of both legs. The 3D position of each marker and whole-body video footage were collected simultaneously and synchronously using four cameras. A control object was used for calibration $(120 \times 60 \times 50 \mathrm{~cm}$ frame). Data were recorded at a sampling frequency of $60 \mathrm{~Hz}$ (Figure 2).

Data acquisition and processing were performed using KineAnalyzer ${ }^{\mathrm{TM}}$ software version 4.0 (Kissei Comtec Co., Ltd., Nagano, Japan) accompanying the device. To detect the timing of foot contact (FC) and foot off (FO) quickly and accurately, we took two steps to decide FC-time and FO-time. First, the rough FC-time and FO-time were automatically estimated based on the motion trajectories of the markers. In this system, the rough FC-time was defined as the time point when the distance between the markers of the lateral malleoli on both legs becomes longest in the anterior-posterior direction. The rough FO-time was defined as the time point when the angle between the lower leg (i.e., the line connecting knee joint marker with the lateral malleoli marker) and the vertical line reaches its maximum in the sagittal plane. Second, to adjust estimated time points to accurate points, two researchers who were experts in motion analysis checked and adjusted the timing carefully frame-by-frame using clear whole-body video footage. ${ }^{[23]}$ The FC-position and FO-position were calculated using the motion capture data of the markers. The origin of the reference system was located at the midpoint between the most anterior position and most posterior position of the lateral malleolus during walking.

\section{Statistical analysis}

Independent t-test was used to test the homogeneity of the general characteristics

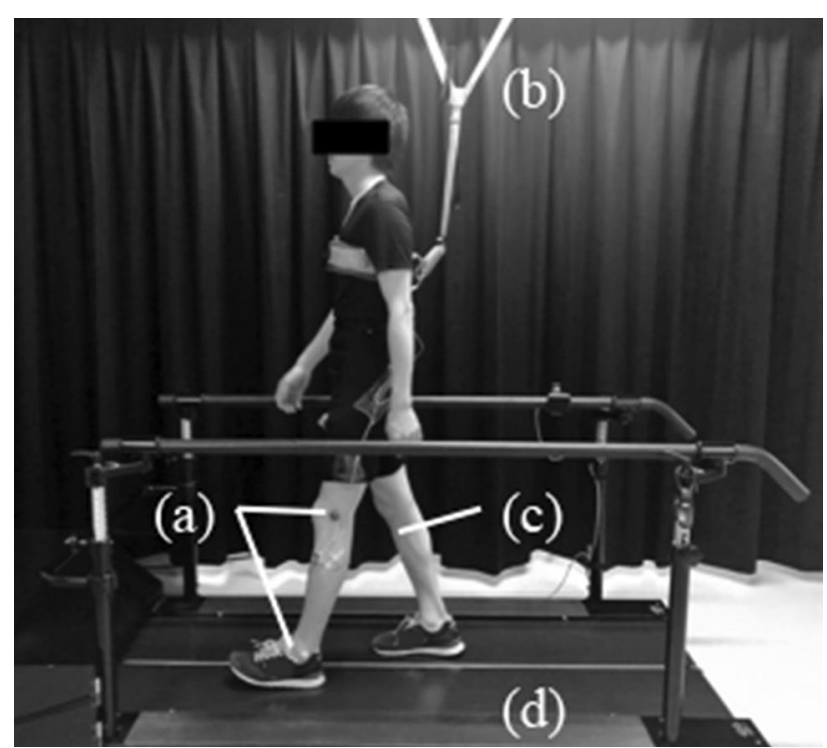

Figure 2. Experimental setup.

(a) Optical markers of $30 \mathrm{~mm}$; (b) Harness; (c) Surface electrode; (d) Split-belt treadmill. 
(age, height, body weight, body mass index) between the groups. The electromyographic signal of TA was smoothed by the root mean square and normalized by dividing by the MVIC (MVIC\%). Next, we extracted one gait cycle obtained in the measurement interval of $15 \mathrm{sec}$, full-wave rectification and normalized the time axis to one gait cycle as $100 \%$, and signal-averaged. Based on the method of Ogawa et al., ${ }^{[17]}$ the stance phase was divided in half from the added average waveform of one gait cycle, and the average amplitude in the first half of stance was calculated as the TA muscle activity. We used a two-way mixed analysis of variance to assess the effects of ankle fixation (fixation group or control group) as betweenfactors and split-belt treadmill walking period (early phase or late phase) as within-factors. All statistical analysis was performed with IBM SPSS version 24.0 (IBM Corp., Armonk, NY, USA), and the significance probability was set to $\mathrm{p}<0.05$.

\section{RESULTS}

The general characteristics of the participants in this study are shown in Table 1 . There were no significant differences in the general characteristics (age, height, body weight, body mass index) between the groups ( $\mathrm{p}>0.05)$.

Figure 3 shows the early phase and late phase EMG of split-belt walking in each group. The TA muscle activity decreased in the late phase regardless of ankle joint fixation. It also decreased in the fixation group regardless of the time periods. Figure 4 shows the amount of change of TA muscle during adaptation period. The results showed a significant main effect for both time period and ankle joint fixation (time period: $\mathrm{F}=802.4, \quad \mathrm{p}<0.001$, ankle joint fixation: $\mathrm{F}=113.7$, $\mathrm{p}<0.001)$. Furthermore, a significant interaction between these factors was also observed $(\mathrm{f}=78.5$, $\mathrm{p}<0.001)$. These results indicated that the degree of decrease in the TA muscle activity during split-belt treadmill walking changes with ankle fixation.

\begin{tabular}{|c|c|c|c|}
\hline \multicolumn{4}{|c|}{$\begin{array}{c}\text { TABLE } 1 \\
\text { General characteristics of participants }\end{array}$} \\
\hline & Fixation group & Control group & \\
\hline & Mean \pm SD & Mean \pm SD & $p$ \\
\hline Age (year) & $30.1 \pm 6.6$ & $32.7 \pm 14.5$ & 0.679 \\
\hline Height $(\mathrm{cm})$ & $174.1 \pm 5.1$ & $174.1 \pm 5.8$ & 0.981 \\
\hline Body weight (kg) & $67.4 \pm 14.4$ & $62.0 \pm 5.8$ & 0.390 \\
\hline Body mass index $\left(\mathrm{kg} / \mathrm{m}^{2}\right)$ & $22.1 \pm 3.5$ & $20.4 \pm 1.4$ & 0.285 \\
\hline
\end{tabular}

(a) Fixation group

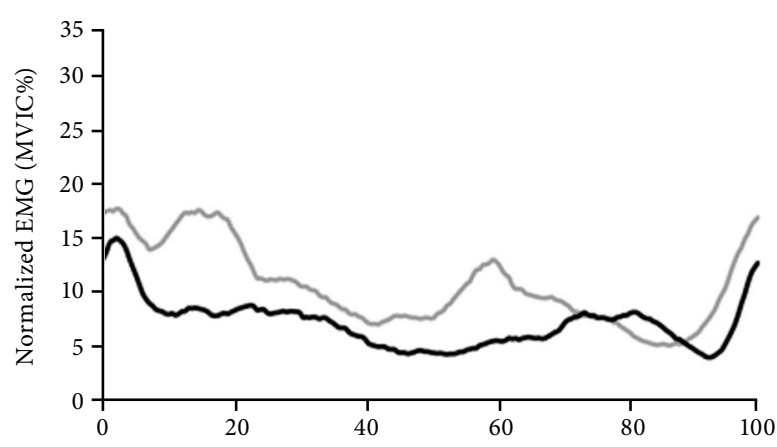

(b) Control group

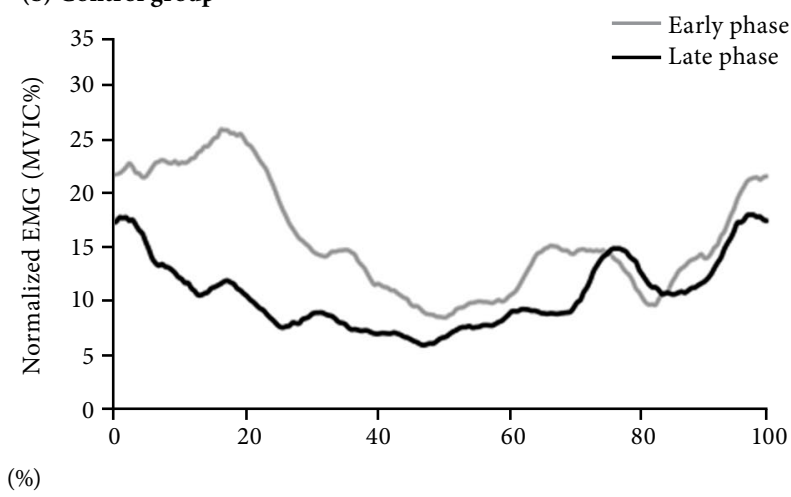

Figure 3. Electromyography of split-belt walking in adaptation period. Figures show average electromyographic activity for tibialis anterior muscle during walking on split-belt treadmill at each condition. Left figure shows fixation group that walked with ankle-foot orthosis for immobilization of right ankle. Right figure shows control group. Gray line indicates early phase, whereas black line indicates late phase.

EMG: Electromyography; MVIC: Maximum voluntary isometric contraction. 


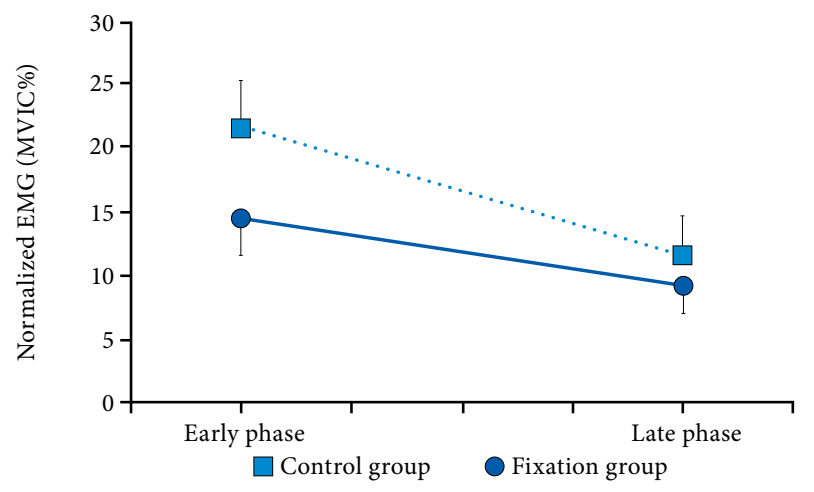

Figure 4. Amount of change of tibialis anterior muscle during adaptation period. Solid line and filled circle indicate fixation group, and dotted line and open square indicate control group. Horizontal axis indicates each phase. Vertical axis indicates electromyographic activities of tibialis anterior muscle. Electromyographic activity for tibialis anterior muscle was changed between presence or absence of ankle fixation and phase of adaptation period.

EMG: Electromyography; MVIC: Maximum voluntary isometric contraction.

\section{DISCUSSION}

In this study, we demonstrated the following three findings. First, the TA muscle activity decreased significantly in the late phase of split-belt walking, regardless of ankle joint fixation. Second, the TA muscle activity in the fixation group was significantly lower than that in the control group regardless of whether it was before or after adaptation. Third, a significant interaction between ankle joint fixation (presence/absence) and time period (early phase and late phase) was observed.

These results are consistent with those of previous studies, which have shown that the TA muscle activity is reduced in the late phase of split-belt walking. Specifically, the TA muscle activity increased on the slow belt side during the early phase of asymmetric walking and subsequently decreased as walking adaptation progressed. ${ }^{[17]}$ To maintain balance during the long swing phase on the fast belt, TA continued to be active during most of the stance phase on the slow belt. ${ }^{[24]}$ We believe that this is one of the reasons for increased TA muscle activity on the slow belt during the early phase of split-belt walking.

The ankle-fixed group had less TA muscle activity compared with the control group because orthoses compensated for the heel locker function of the FC for the point where the TA muscle activity was lower in the fixation group. Healthy subjects are braked by footfall mainly due to the eccentric contraction of the TA muscle from the heel contact period to the plantar contact period of the gait cycle. ${ }^{[18]}$ However, because ankle-foot orthosis limits dorsiflexion of the ankle joint, the foot and lower leg are integrated at heel contact to the sole contact period and thus rotate forward-center to the heel. ${ }^{[25]}$

Although the TA muscle activity in the split-belt late phase decreased in the ankle fixation group as well, the TA muscle activity in the first half stance was adapted by compensating with the ankle-foot orthosis, and thus, the degree of decrease was smaller than that in the control group. In addition, smaller adjustment at the foot level might be explained by the fact that the heel locker function is constant owing to the fixed ankle-foot orthosis.

A limitation of this study was the young age of the participants. Because post-stroke patients are generally elderly, it will be necessary to select participants considering their age.

In conclusion, the TA muscle activity was decreased significantly in the latter phase of split-belt walking, regardless of ankle joint fixation. However, the TA muscle activity significantly reduced with ankle fixation, regardless of the split-belt walking time. These data show that the TA muscle activity adaptation is reduced when the ankle joint is fixed, which suggests that orthoses, which fix ankle joint, would reduce adaptation to split-belt treadmill walking.

\section{Acknowledgments}

Sincere appreciation is extended to all participants in this study.

\section{Declaration of conflicting interests}

The authors declared no conflicts of interest with respect to the authorship and/or publication of this article.

Funding

The authors received no financial support for the research and/or authorship of this article.

\section{REFERENCES}

1. Takashima N, Arima H, Kita Y, Fujii T, Miyamatsu N, Komori M, et al. Incidence, Management and Short-Term Outcome of Stroke in a General Population of 1.4 Million Japanese - Shiga Stroke Registry. Circ J 2017;81:1636-46.

2. Schmid A, Duncan PW, Studenski S, Lai SM, Richards L, Perera S, et al. Improvements in speed-based gait classifications are meaningful. Stroke 2007;38:2096-100.

3. Laufer Y, Dickstein R, Chefez Y, Marcovitz E. The effect of treadmill training on the ambulation of stroke survivors in the early stages of rehabilitation: a randomized study. J Rehabil Res Dev 2001;38:69-78. 
4. Ada L, Dean CM, Hall JM, Bampton J, Crompton S. A treadmill and overground walking program improves walking in persons residing in the community after stroke: a placebo-controlled, randomized trial. Arch Phys Med Rehabil 2003;84:1486-91.

5. Hesse S, Bertelt C, Schaffrin A, Malezic M, Mauritz KH. Restoration of gait in nonambulatory hemiparetic patients by treadmill training with partial body-weight support. Arch Phys Med Rehabil 1994;75:1087-93.

6. da Silva RS, da Silva ST, de Souza JM, de Figueiredo MCC, Mendes TAS, de Sena Nunes MC, et al. Effects of inclined treadmill training on functional and cardiovascular parameters of stroke patients: study protocol for a randomized controlled trial. Trials 2019;20:252.

7. Madhavan S, Lim H, Sivaramakrishnan A, Iyer P. Effects of high intensity speed-based treadmill training on ambulatory function in people with chronic stroke: A preliminary study with long-term follow-up. Sci Rep 2019;9:1985.

8. Wall JC, Turnbull GI. Gait asymmetries in residual hemiplegia. Arch Phys Med Rehabil 1986;67:550-3.

9. Chen G, Patten C, Kothari DH, Zajac FE. Gait differences between individuals with post-stroke hemiparesis and non-disabled controls at matched speeds. Gait Posture 2005;22:51-6.

10. Saito E, Ohtsuka K. Gait analysis and motion analysis. Yayoi: Center for Academic Publications Japan; 2015. p. 121 [Article in Japanese]

11. Hsu AL, Tang PF, Jan MH. Analysis of impairments influencing gait velocity and asymmetry of hemiplegic patients after mild to moderate stroke. Arch Phys Med Rehabil 2003;84:1185-93.

12. Bovonsunthonchai S, Hiengkaew V, Vachalathiti R, Vongsirinavarat M. Gait symmetrical indexes and their relationships to muscle tone, lower extremity function, and postural balance in mild to moderate stroke. J Med Assoc Thai 2011;94:476-84.

13. Balasubramanian CK, Bowden MG, Neptune RR, Kautz SA. Relationship between step length asymmetry and walking performance in subjects with chronic hemiparesis. Arch Phys Med Rehabil 2007;88:43-9.

14. Reisman DS, Wityk R, Silver K, Bastian AJ. Locomotor adaptation on a split-belt treadmill can improve walking symmetry post-stroke. Brain 2007;130:1861-72.
15. Reisman DS, McLean H, Bastian AJ. Split-belt treadmill training poststroke: a case study. J Neurol Phys Ther 2010;34:202-7.

16. Reisman DS, McLean H, Keller J, Danks KA, Bastian AJ. Repeated split-belt treadmill training improves poststroke step length asymmetry. Neurorehabil Neural Repair 2013;27:460-8.

17. Ogawa T, Kawashima N, Ogata T, Nakazawa K. Predictive control of ankle stiffness at heel contact is a key element of locomotor adaptation during split-belt treadmill walking in humans. J Neurophysiol 2014;111:722-32.

18. Perry J, Burnfield JM, editors. Gait Analysis: Normal and Pathological Function. 2nd ed. Thorofare: SLACK Incorporated; 2010.

19. Eftekhari N. Amputation rehabilitation: In: O'Young B, Young MA, Stiens SA, editors. PMR Secrets. Philadelphia: Hanley \& Belfus Inc.; 1999. p. 214-22.

20. Hislop HJ, Avers D, Brown M, editors. Daniels and Worthingham's Muscle Testing: Techniques of Manual Examination and Performance Testing. 9th ed. St. Louis: Elsevier; 2013. p. 261.

21. Lauzière S, Miéville $C$, Betschart M, Duclos C, Aissaoui $\mathrm{R}$, Nadeau S. Plantarflexion moment is a contributor to step length after-effect following walking on a split-belt treadmill in individuals with stroke and healthy individuals. J Rehabil Med 2014;46:849-57.

22. Vasudevan EV, Torres-Oviedo G, Morton SM, Yang JF, Bastian AJ. Younger is not always better: development of locomotor adaptation from childhood to adulthood. J Neurosci 2011;31:3055-65.

23. Tanikawa $H$, Ohtsuka $\mathrm{K}$, Mukaino M, Inagaki K, Matsuda F, Teranishi T, et al. Quantitative assessment of retropulsion of the hip, excessive hip external rotation, and excessive lateral shift of the trunk over the unaffected side in hemiplegia using three-dimensional treadmill gait analysis. Top Stroke Rehabil 2016;23:311-7.

24. Duysens J, Bastiaanse CM, Smits-Engelsman BC, Dietz V. Gait acts as a gate for reflexes from the foot. Can J Physiol Pharmacol 2004;82:715-22.

25. Isokane N, Kimura Y, Kondo Y, Sassa H, Hashimoto K, Hayashi S et al. Tankashisogu no kakato no katasa ga hoko ni ataeru eikyo. [Article in Japanese] Chubu Rehabilitation College 2011;6:38-41. 